\title{
Solar Diurnal and Semi-Diurnal Variations of Cosmic Rays Observed by the North-South Network of Surface-Level Multi-Directional Muon Telescopes
}

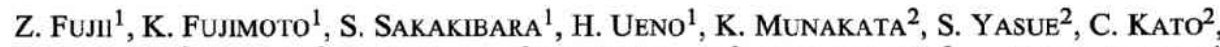

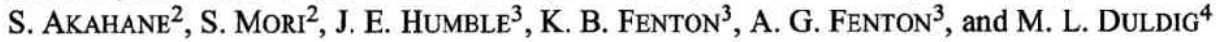 \\ ${ }^{1}$ Solar-Terrestrial Environment Laboratory, Nagoya University, Nagoya 464-01, Japan \\ ${ }^{2}$ Department of Physics, Faculty of Science, Shinshu University, Matsumoto 390, Japan \\ ${ }^{3}$ Physics Department, University of Tasmania, Hobart, Tasmania 7001, Australia \\ ${ }^{4}$ Australian Antarctic Division, Kingston, Tasmania 7150, Australia
}

(Received December 5, 1994; Accepted March 9, 1995)

\begin{abstract}
A scintillator muon telescope was installed at the Hobart Campus of the University of Tasmania $\left(42.85^{\circ} \mathrm{S}, 147.42^{\circ} \mathrm{E}\right)$, Australia in December 1991. It is part of a North-South Network of MultiDirectional Cosmic Ray Muon Surface Telescopes, in conjunction with the Nagoya Cosmic Ray Muon Telescope $\left(35.12^{\circ} \mathrm{N}, 136.97^{\circ} \mathrm{E}\right)$. Using two years of data from the North-South Network, we have investigated the average features of the solar diurnal and semi-diurnal variations. Preliminary results of the analysis show that two years averages of the observed diurnal variations are composed of a northsouth symmetric and an asymmetric variations. The north-south asymmetric diurnal variation is consistent with that expected from the semi-diurnal anisotropy which produces the observed semi-diurnal variation. It is shown that the reference axis of the anisotropy is in the direction of about $7^{\circ} \mathrm{S}$ sunward from the ecliptic.
\end{abstract}

\section{Introduction}

Studies of solar daily variations of cosmic rays provide valuable information on the physical process of cosmic ray modulation in the heliosphere. Nagashima (1971) and Nagashima and Ueno (1971) formulated a model of the cosmic ray daily variations arising from a generalized axis-symmetric anisotropy, to give the basis of analysis in the three dimensional space. In this framework, many investigations have been made of the origin of the solar semi-diurnal variation, and it was shown that the observed semi-diurnal variation can be interpreted by the pitch angle distribution $\left(F_{2}(X) \propto \sin ^{2} X\right)$ of $\operatorname{cosmic}$ ray particles about the interplanetary magnetic field line (Nagashima et al., 1972; Fujii, 1972). This second space distribution also produce a special diurnal anisotropy represented by a spherical $P_{2}{ }^{1}(\cos \theta)$-type function whose latitudinal dependence is north-south asymmetric. On the other hand, it is known that there exists the diurnal variation due to the so called co-rotational anisotropy with a relatively large amplitude. Observed diurnal variations are composed of these two space harmonic components which have different latitudinal dependences (Fujimoto et al., 1973). To study these diurnal variations, it is important to analyse simultaneously data observed in both hemisphere. In this report, we present briefly the solar diurnal and semi-diurnal variations observed by the North-South Network of Surface-Level Multi-Directional Muon Telescopes.

\section{The North-South Network of Surface-Level Multi-Directional Muon Telescopes}

A scintillator muon telescope was installed at the Hobart Campus of the University of Tasmania $\left(42.85^{\circ} \mathrm{S}, 147.42^{\circ} \mathrm{E}\right.$, sea level), Australia, in December 1991. It is part of a North-South Network of MultiDirectional Cosmic Ray Surface Muon Telescope, in conjunction with the Nagoya cosmic ray muon 
telescope $\left(35.12^{\circ} \mathrm{N}, 136.97^{\circ} \mathrm{E}\right.$, Sekido et al., 1975) which have been in operation since 1970 . The Hobart telescope are of similar design of those at Nagoya and telescope consist of two layers of $3 \mathrm{~m} \times 3 \mathrm{~m}$, onequarter of Nagoya telescopes in detector area. The telescopes record 13 different directional intensities, taking coincidence between pulses from a specified pair of detectors of $1 \mathrm{~m} \times 1 \mathrm{~m}$ in the upper and lower layers. The asymptotic directions of five directional telescopes, Vertical, $30^{\circ} \mathrm{North}, 30^{\circ} \mathrm{South}, 30^{\circ}$ East and $30^{\circ} \mathrm{West}$ are shown in Fig. 1, together with those of the Nagoya telescopes. Characteristics of the Hobart and Nagoya telescopes are listed in Table 1 (comp.: Component telescope, $\lambda_{\mathrm{E}}$ : declination of effective viewing direction, $P_{\mathrm{c}}$ : geomagnetic cutoff rigidity, $I$ : hourly counting rate, $P_{\mathrm{m}}$ : effective median rigidity).
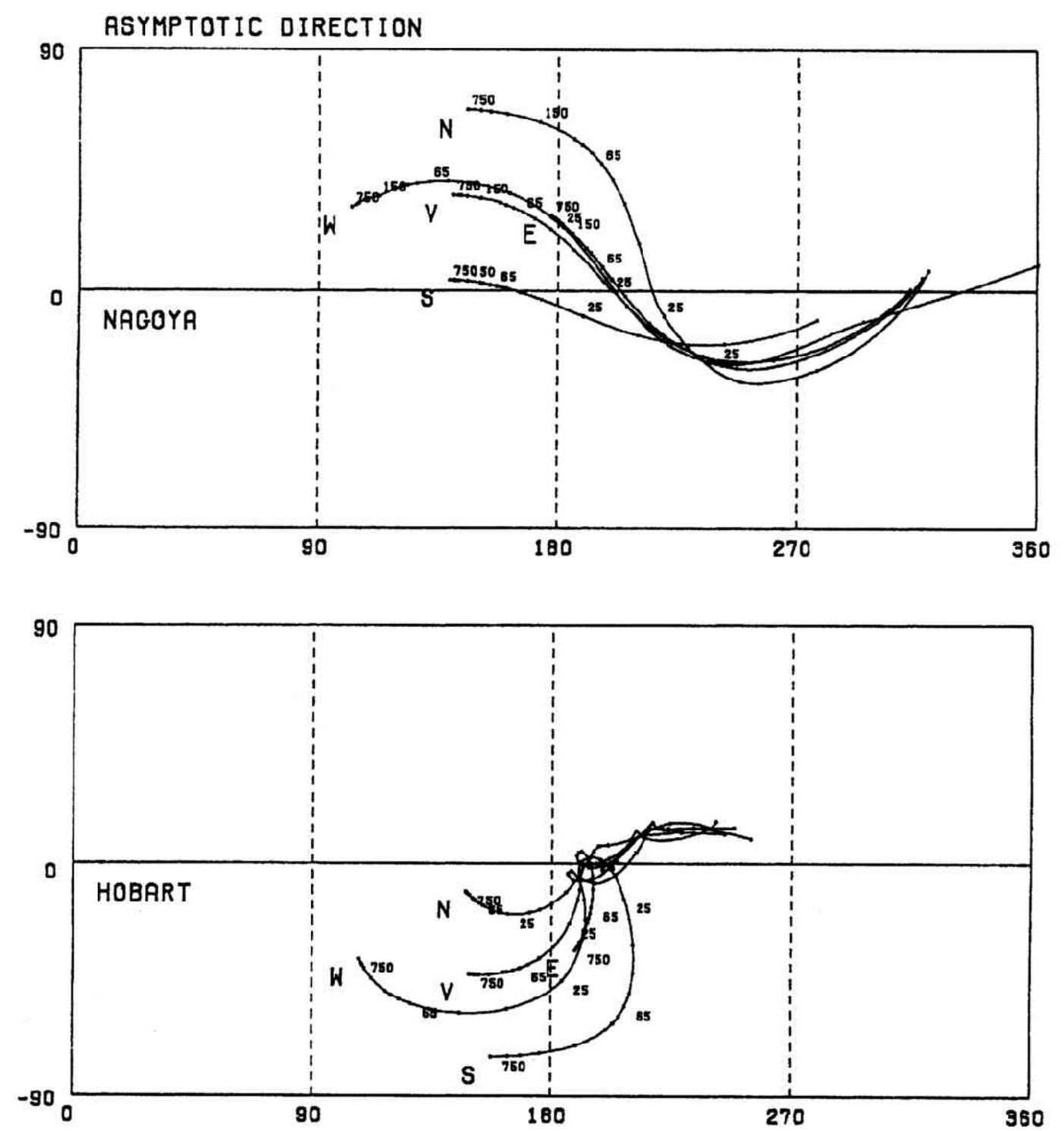

Fig. 1. The asymptotic orbital direction of the cosmic rays with the rigidity $P$ incident parallel to each telescope at Nagoya (top) and Hobart (bottom), its direction $(V, E, \ldots)$ being schematically shown in Fig. 1. The axis of ordinates and abscissas show the geographic latitude and the geographic longitude in degree, respectively. 
Table 1. Characteristics of the Nagoya and Hobart Multi-Directional Surface Muon Telescopes.

Nagoya Scintillator Telescope

\begin{tabular}{lrrccc}
\hline Comp. & \multicolumn{1}{c}{$\lambda_{\mathrm{E}}$} & $P_{\mathrm{c}}(\mathrm{GV})$ & $I_{\text {obs }}\left(10^{4}\right.$ c.p.h. $)$ & $I_{\text {cal }}\left(10^{4}\right.$ c.p.h. $)$ & $P_{\mathrm{m}}(\mathrm{GV})$ \\
\hline $\mathrm{V}$ & $18.0^{\circ} \mathrm{N}$ & 11.5 & 276 & 243 & 60.0 \\
$\mathrm{~N}$ & $38.0^{\circ} \mathrm{N}$ & 12.9 & 125 & 110 & 66.0 \\
$\mathrm{~S}$ & $4.0^{\circ} \mathrm{N}$ & 11.3 & 123 & 112 & 64.0 \\
$\mathrm{E}$ & $9.0^{\circ} \mathrm{N}$ & 16.2 & 120 & 108 & 67.0 \\
$\mathrm{~W}$ & $25.0^{\circ} \mathrm{N}$ & 9.4 & 126 & 113 & 63.0 \\
$\mathrm{NE}$ & $19.0^{\circ} \mathrm{N}$ & 17.6 & 58.0 & 52.0 & 73.0 \\
$\mathrm{NW}$ & $37.0^{\circ} \mathrm{N}$ & 11.0 & 62.0 & 54.4 & 68.0 \\
$\mathrm{SE}$ & $2.0^{\circ} \mathrm{S}$ & 15.0 & 58.0 & 53.1 & 71.0 \\
$\mathrm{SW}$ & $11.0^{\circ} \mathrm{N}$ & 9.2 & 60.0 & 54.9 & 67.0 \\
$\mathrm{~N} 2$ & $33.0^{\circ} \mathrm{N}$ & 12.9 & 61.1 & 53.8 & 87.0 \\
$\mathrm{~S} 2$ & $6.0^{\circ} \mathrm{S}$ & 10.9 & 59.9 & 54.6 & 85.0 \\
$\mathrm{E} 2$ & $1.0^{\circ} \mathrm{N}$ & 21.0 & 58.1 & 52.1 & 91.0 \\
W2 & $27.0^{\circ} \mathrm{N}$ & 9.3 & 61.7 & 55.2 & 84.0 \\
$\mathrm{~N} 3$ & $36.0^{\circ} \mathrm{N}$ & 11.0 & 17.8 & 13.4 & 112.0 \\
$\mathrm{~S} 3$ & $18.0^{\circ} \mathrm{S}$ & 10.8 & 17.6 & 13.5 & 111.0 \\
$\mathrm{E} 3$ & $3.0^{\circ} \mathrm{S}$ & 25.1 & 17.3 & 12.9 & 119.0 \\
W3 & $36.0^{\circ} \mathrm{N}$ & 8.7 & 18.0 & 13.6 & 110.0 \\
\hline
\end{tabular}

Hobart Scintillator Telescope

\begin{tabular}{lrcccc}
\hline Comp. & \multicolumn{1}{c}{$\lambda_{\mathrm{E}}$} & $P_{\mathrm{c}}(\mathrm{GV})$ & $I_{\text {obs }}\left(10^{4}\right.$ c.p.h. $)$ & $I_{\text {cal }}\left(10^{4}\right.$ c.p.h. $)$ & $P_{\mathrm{m}}(\mathrm{GV})$ \\
\hline $\mathrm{V}$ & $30.0^{\circ} \mathrm{S}$ & 2.5 & 89 & 64 & 56.4 \\
$\mathrm{~N}$ & $19.0^{\circ} \mathrm{S}$ & 2.5 & 32 & 23 & 61.6 \\
$\mathrm{~S}$ & $37.6^{\circ} \mathrm{S}$ & 2.5 & 33 & 23 & 61.6 \\
$\mathrm{E}$ & $20.0^{\circ} \mathrm{S}$ & 2.5 & 33 & 23 & 61.6 \\
$\mathrm{~W}$ & $386^{\circ} \mathrm{S}$ & 2.5 & 32 & 23 & 61.6 \\
$\mathrm{NE}$ & $9.4^{\circ} \mathrm{S}$ & 2.5 & 12 & 9.0 & 66.8 \\
$\mathrm{NW}$ & $26.3^{\circ} \mathrm{S}$ & 2.5 & 12 & 9.0 & 66.8 \\
$\mathrm{SE}$ & $26.1^{\circ} \mathrm{S}$ & 2.5 & 13 & 9.0 & 66.8 \\
$\mathrm{SW}$ & $468^{\circ} \mathrm{S}$ & 2.5 & 13 & 9.0 & 66.8 \\
$\mathrm{~N} 2$ & $6.0^{\circ} \mathrm{S}$ & 2.5 & 8.7 & 5.6 & 81.1 \\
$\mathrm{~S} 2$ & $40.0^{\circ} \mathrm{S}$ & 2.5 & 9.4 & 5.6 & 81.1 \\
$\mathrm{E} 2$ & $8.1^{\circ} \mathrm{S}$ & 2.5 & 8.9 & 5.6 & 81.1 \\
$\mathrm{~W} 2$ & $39.8^{\circ} \mathrm{S}$ & 2.5 & 8.6 & 5.6 & 81.1 \\
\hline
\end{tabular}

\section{Observed Solar Diurnal and Semi-Diurnal Variations}

The Hobart muon telescope started continous observations of cosmic ray intensities in late December, 1991. More than two years of data are now available from the North-South Network of surfacelevel muon telescopes. Observed hourly value intensities are corrected for pressure changes, and analysed by harmonic analysis to derive the diurnal and semi-diurnal harmonic coefficients on a daily basis. The obtained diurnal vectors are corrected for Compton-Getting effect due to the earth's orbital motion around the sun, and the diurnal and semi-diurnal vectors are averaged for the observed period of two complete years of 1992 and 1993. The mean vectors thus derived for Nagoya and Hobart are shown in harmonic dial in Fig. 2. In the figure, the end-points of vectors are connected by solid and dotted lines, to show the relative relation of amplitudes and phases observed by the directional telescopes. 

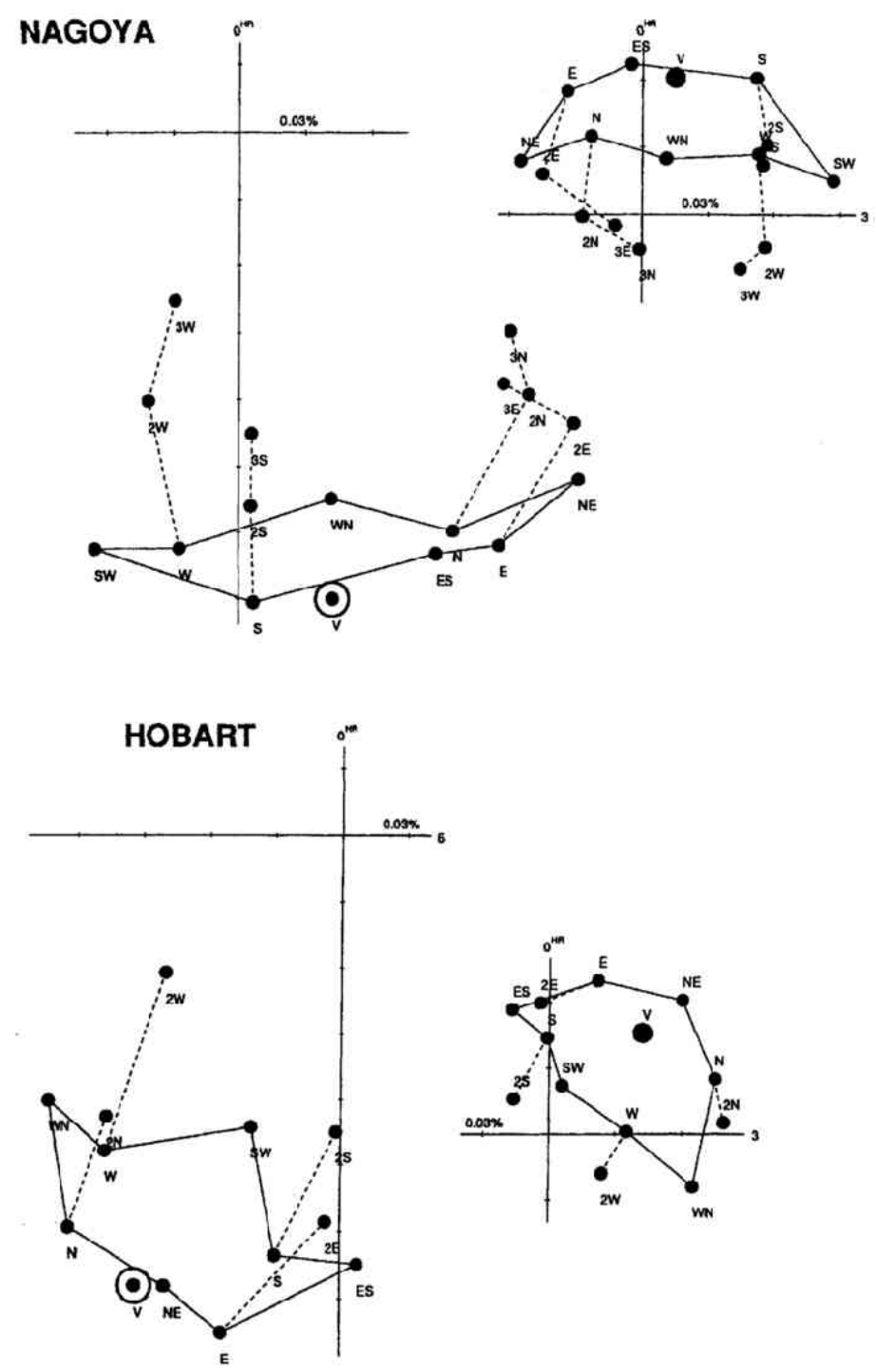

Fig. 2. Average solar diurnal and semi-diurnal variations observed with 17 and 13 directional muon telescopes at Nagoya and Hobart, respectively. Symbol expresses the directional telescope (cf. Table 1).

\section{Analysis}

The axis-symmetric distribution is expressed by the following equation,

$$
\frac{J(X, P)}{J(P)}=F(X) G(P)=G(P) \sum_{n=0}^{\infty} F_{n}(X)=G(P) \sum_{n=0}^{\infty} \eta_{n} P_{n}(\cos X)
$$

where $P$ is the cosmic ray rigidity, $G(P)$ is the differential rigidity dependence of the anisotropy and $X$ defines the cosmic ray incident direction (OJ) relative to the reference axis of the anisotropy (OR) as shown in Fig. 3. The $m$-th space distribution $F_{n}(X)$ is transformed into the projected component $f_{n}^{m}(X)$ as 


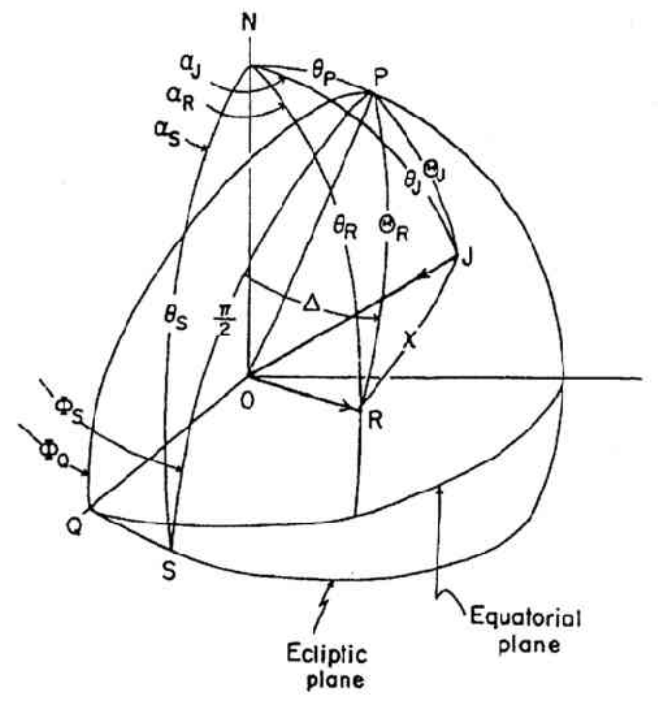

Fig. 3. Equatorial and ecliptic coordinates systems. N: Equatorial north pole, P: Ecliptic north pole.

$$
F_{n}(X)=\sum_{n=0}^{\infty} f_{n}^{m}(X)=\sum_{n=0}^{\infty} \eta_{n} P_{n}^{m}\left(\cos \theta_{\mathrm{J}}\right) P_{n}^{m}\left(\cos \theta_{\mathrm{R}}\right) \cos m\left(\alpha_{\mathrm{J}}-\alpha_{\mathrm{R}}\right)
$$

where $\left(\theta_{\mathrm{J}}, \alpha_{\mathrm{J}}\right)$ and $\left(\theta_{\mathrm{R}}, \alpha_{\mathrm{R}}\right)$ represent the direction of the cosmic rays and the reference axis in equatorial coordinate respectively. The $m$-th harmonic component $D_{n}{ }^{m}(t)$ of the daily variation at a station is related to $f_{n}^{m}(X)$ and $G(P)$ as

$$
\begin{gathered}
D_{n}^{m}(t)=A_{n}^{m} \cos \frac{2 m \pi t}{24}+B_{n}^{m} \sin \frac{2 m \pi t}{24} \\
\left(\begin{array}{c}
A_{n}^{m} \\
B_{n}^{m}
\end{array}\right)=\left(\begin{array}{cc}
c_{n}^{m} & s_{n}^{m} \\
-s_{n}^{m} & c_{n}^{m}
\end{array}\right)\left(\begin{array}{l}
x_{n}^{m} \\
y_{n}^{m}
\end{array}\right) \quad\left(\begin{array}{l}
x_{n}^{m} \\
y_{n}^{m}
\end{array}\right)=\eta_{n} P_{n}^{m}\left(\cos \theta_{\mathrm{R}}\right)\left(\begin{array}{l}
\cos \frac{2 m \pi t}{24} t_{\mathrm{R}} \\
\sin \frac{2 m \pi t}{24} t_{\mathrm{R}}
\end{array}\right)
\end{gathered}
$$

where $t_{\mathrm{R}}$ is the local time of the reference axis, and $c_{n}{ }^{m}$ and $s_{n}{ }^{m}$ are the coupling coefficients. The harmonic components, $D_{1}{ }^{1}$ and $D_{2}^{2}$ are calculated for Hobart, using the coupling coefficients, and are shown in Fig. 4 for the typical rigidity dependences of the diurnal and semi-diurnal variations. It should be noted that the observed semi-diurnal vectors shown in Fig. 2 are quite similar to the configuration of the calculated $D_{2}^{2}$. On the other hand, the observed diurnal vectors have the configuration deformed from that of the calculated $D_{1}^{1}$, suggesting a contribution by the harmonic component $D_{2}{ }^{1}$. In the following, we assumed a power law rigidity dependence of $P^{\gamma /}$ with an upper cut-off rigidity $P_{\mathrm{U}}$ for $G(P)$. The semi-diurnal anisotropy was first determined by the best fit between the observed and the expected values. The obtained amplitude of the semi-diurnal anisotropy is $0.100 \pm 0.003 \%$ at $10 \mathrm{GV}$ with $P_{\mathrm{U}}=100 \mathrm{GV}$ and $\gamma=0.5$, and the phase is 3.05 and $15.05 \pm 0.05 \mathrm{hr}$. Using the rigidity dependence of $D_{2}{ }^{2}$ for $D_{2}{ }^{1}$, the observed diurnal variations are analysed, assuming the existence of two harmonic components $D_{1}{ }^{1}$ and $D_{2}{ }^{1}$. The obtained amplitudes and the phases of $D_{1}^{1}$ in space are $0.37 \pm 0.1 \%$ and $15.3 \mathrm{hr}$ with $P_{\mathrm{U}}=100 \mathrm{GV}$ and $\gamma=0$, and those of $D_{2}{ }^{1}$ are $0.023 \pm 0.007 \%, 15.3 \pm 1.3$. It should be pointed out here that the obtained phase of $D_{2}{ }^{1}$ 


\section{HOBART}
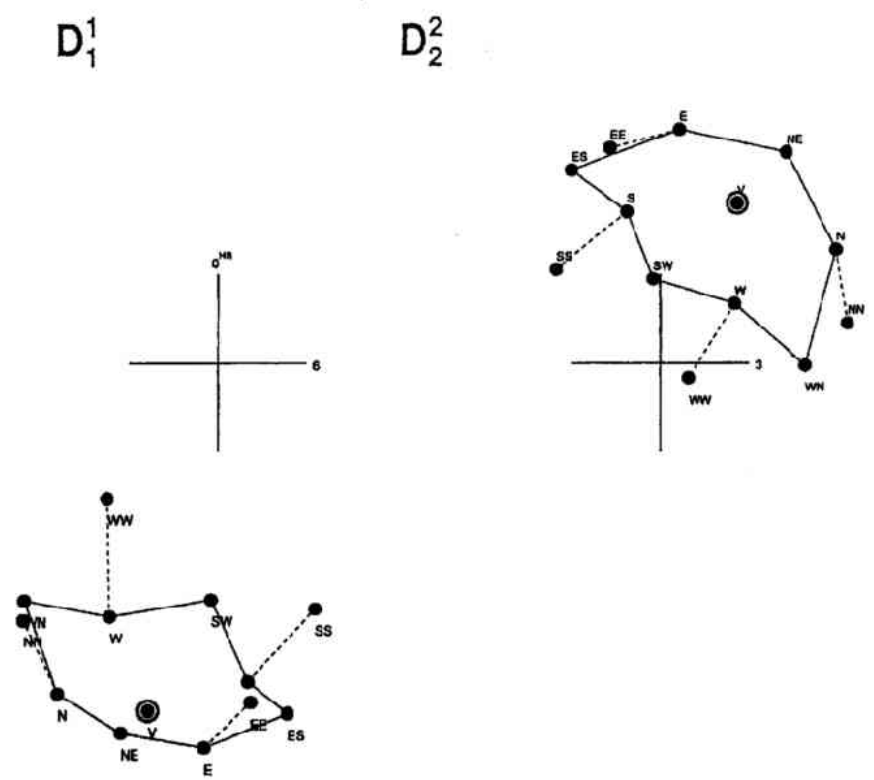

Fig. 4. $D_{1}{ }^{1}$ and $D_{2}{ }^{2}$ calculated for Hobart telescope. The phase and the rigidity dependence are assumed to be $15 \mathrm{hr}$ and $P^{0}$ with $P_{\mathrm{U}}=100 \mathrm{GV}$ for $D_{1}{ }^{1}$, and $3 \mathrm{hr}$ and $P^{0.5}$ with $P_{\mathrm{U}}=100 \mathrm{GV}$ for $D_{2}{ }^{2}$ respectively.

coincides with that of $D_{2}^{2}$ within the accuracy of the observations. This implies that $D_{2}{ }^{1}$ and $D_{2}{ }^{2}$ are produced by the pitch angle distribution of $F_{2}(X)$ whose reference axis is almost parallel to the average direction of the interplanetary magnetic field. The ratio of the obtained amplitudes indicates that the average reference axis of the anisotropy is in the direction of about $7^{\circ} \mathrm{S}$ sunward from the ecliptic.

In summary, we have investigated the average features of the solar diurnal and semi-diurnal variations, using two years of data from the North-South Network of Multi-Directional Cosmic Ray Surface Telescopes. It is shown that the observed diurnal variations are composed of a north-south symmetric variation and an asymmetric variation. Preliminary results of analysis indicate that the reference axis of the semi-diurnal anisotropy is in the direction of about $7^{\circ} \mathrm{S}$ sunward from the ecliptic.

\section{REFERENCES}

Fujii, Z., The solar semi-diurnal anisotropy of the cosmic radiation, Rep. Ionos. Space Res. Japan, 25, No, 3, $242-270,1971$. Fujimoto, K., K. Nagashima, H. Ueno, and I. Kondo, Three dimensional cosmic ray anisotropy in interplanetary space responsible for solar semi-diurnal variation, 13th Intern. Conf. Cosmic Rays, Denver, 2, 993-998, 1973.

Nagashima, K., Three-dimensional cosmic ray anisotropy in interplanetary space, Part I. Formulation of cosmic ray daily variation produced by axis-symmetric anisotropy, Rep. Ionos. Space Res. Japan, 25, No. 3, 189-211, 1971.

Nagashima, K. and H. Ueno, Three-dimensional cosmic ray anisotropy in interplanetary space, Part II. General expression of annual modulation of daily variation by frequency modulation method and its application to the modulation due to earth's revolution around sun, Rep. Ionos. Space Res. Japan, 25, No. 3, 212-241, 1971.

Nagashima, K., H. Ueno, K. Fujimoto, Z. Fujii, and I. Kondo, Three-dimensional cosmic ray anisotropy in interplanetary space, Part III. Origin of cosmic ray solar semi-diurnal variation, Rep. Ionos. Space Res. Japan, 26, No. 1/2, 1-30, 1972.

Sekido, Y., K. Nagashima, I. Kondo, H. Ueno, K. Fujimoto, and Z. Fujii, Rep. of Cosmic-Ray Res. Lab., No. 2, 1975. 